GRADIATION\&APPLICATIONS

ISSN 2466-4294 (online) | rad-journal.org

Vol. 2 | Issue 3 | pp. $169-174,2017$

doi: 10.21175/RadJ.2017.03.035

Original research paper

\title{
CHEMICAL SHIFTS OF X-RAY EMISSION SPECTRA AND EFFECTIVE STATES OF YTTERBIUM IN FLUORIDES: EMBEDDED CLUSTER MODELING OF YbF ${ }_{2}$ AND YbF $_{3}$ CRYSTALS* $^{*}$
}

\author{
V.M. Shakhova1,2**, Yu.V. Lomachuk ${ }^{1}$, Yu.A. Demidov', L.V. Skripnikov',2, \\ N.S. Mosyagin' ${ }^{1}$ A.V. Zaitsevskii'1,3, A.V. Titov ${ }^{1,2}$ \\ ${ }^{1}$ B.P. Konstantinov Petersburg Nuclear Physics Institute, Gatchina, Leningrad Region, Russia \\ ${ }^{2}$ Dept. of Physics, Saint Petersburg State University, Saint Petersburg, Russia \\ 3Chemistry Dept., M. Lomonosov Moscow State University, Moscow, Russia
}

\begin{abstract}
The $\mathrm{YbF}_{2}$ and $\mathrm{YbF}_{3}$ crystals were studied within the embedded cluster model. The small core relativistic pseudopotentials for the central $\mathrm{Yb}$ atom (42 valence electrons) and embedding potentials for $\mathrm{Yb}$ and $\mathrm{F}$ atoms were constructed. Chemical shifts of $K_{\alpha 1}$ and $K_{\alpha 2}$ lines of X-ray emission spectra (XES) were calculated using non-variation one-center restoration technique and relativistic density functional theory (relDFT) with the hybrid exchangecorrelation functional PBEO. It was done in the $\mathrm{YbF}_{9} \mathrm{Yb}_{12} \mathrm{~F}_{24}$ cluster simulating the $\mathrm{YbF}_{3}$ crystal with respect to $\mathrm{YbF}_{8} \mathrm{Yb}_{12} \mathrm{~F}_{24}$ one representing the $\mathrm{YbF}_{2}$ crystal. The resulting estimates are $628 \mathrm{meV}$ for $K_{\alpha 1}$ and $559 \mathrm{meV}$ for $\mathrm{K}_{\alpha 2}$ and their weighted mean agrees within $10 \%$ with the experimental value, $557 \pm 27 \mathrm{meV}$. In turn, the weighted relativistic Hartree-Fock (relHF) calculation is higher on $20 \%$. It indicates that the incorporation of electron correlation effects is essential for reproducing the Ka1, 2 chemical shifts.
\end{abstract}

Key words: XES chemical shifts, $K_{\alpha 1}$ and $K_{\alpha 2} X$-ray emission lines, relativistic pseudopotential, relativistic density functional theory, cluster embedded in a crystal

\section{INTRODUCTION}

Ytterbium is one of the lanthanides with favorite valences II and III. The ground state of the ytterbium atom has the electronic configuration [Xe] 4f146s25do. When the $\mathrm{Yb}$ atom is in the divalent state, two electrons from the outermost $6 s$ orbital are involved in the formation of chemical bonds. In its trivalent state, one electron additionally leaves the $4 f$ shell. Such a change of the electronic density in the spatial outercore region (where $4 f$ shell is localized) results in a large chemical shift ( $\mathrm{ChSh}$ ) of the characteristic X-ray emission lines (XES) on Yb. Therefore, the determination of the XES chemical shifts can be an efficient tool for studying changes in the valence states of $d$ and $f$ elements in a chemical compound [1]-[5].

In the present study, the theoretical modeling of the cluster embedded in the $\mathrm{YbF}_{2}$ and $\mathrm{YbF}_{3}$ crystals is carried out and the quantum chemical calculation of XES chemical shifts for $\mathrm{K}_{\alpha_{1}}$ and $\mathrm{K}_{\mathrm{a} 2}$ lines on ytterbium is performed.

\section{PROBLEM}

The XES lines of a heavy atom corresponding to electronic transitions from one inner shell to the other one are characteristic lines for the atom. However, for the same atom in different chemical compounds (or in different valence states), these lines are slightly shifted in relation to each other. The difference between these energies of the inner-core electron transition is known as the XES chemical shift.

The XES chemical shift is a property associated with the spatial core region ("core" property [6]), which is most sensitive to changes of the electron density of valence electrons in the core region. Thus, by studying the chemical shift on an atom, we can estimate the effective valence state of this atom in the compounds under consideration. Therefore, to obtain a reasonable theoretical value of XES chemical shift we need to correctly determine the electronic density near the nucleus under study.

In the present work, the XES ChSh on ytterbium in the $\mathrm{YbF}_{3}$ crystal compared to the $\mathrm{YbF}_{2}$ one are evaluated via simulating the electronic structure of the crystals in the frames of the embedded cluster model with $\mathrm{Yb}$ as a central atom. We consider the clusters in which only the central atom and its nearest neighbors are accurately described (clusters of "minimal size" below) in order to have an opportunity to study them at different levels of correlation treatment, from the density functional theory (DFT) to the wave-function

\footnotetext{
* The paper was presented at the Fifth International Conference on Radiation and Applications in Various Fields of Research (RAD 2017), Budva, Montenegro, 2017.

verahcnkrf@gmail.com
} 
V.M. Shakhova et al., Chemical shifts of x-ray emission spectra..., Rad. Applic., 2017, 2, 3, 169-174

based methods. The main goal of this work is the development of the embedding model for the $\mathrm{YbF}_{2}$ and $\mathrm{YbF}_{3}$ structures for the evaluation of chemical shifts of $\mathrm{K}_{\mathrm{a} 1,2}$ lines.

\section{COMPUTATIONAL DETAILS}

\subsection{Relativistic pseudopotential method}

We employ the relativistic electronic structure model defined by the shape-consistent relativistic pseudopotential (RPP) method [7] for the ytterbium atom. In this paper, the valence version of the generalized RPP, vGRPP [8], [9], [10], is applied, in which the semilocal RPP operator is utilized [11].

The $4 f$ shell in lanthanides is valence by energy, but it is localized in the outer-core spatial region. That leads to either the large number of explicitly treated electrons when using small-core RPP or to essentially lower accuracy for a small number of explicitly treated electrons (large-core RPP versions) in calculations of lanthanide compounds.

The goal of our study is to evaluate the XES chemical shifts, i.e. the property localized on ytterbium atom [4]. For the exact description of this property of ytterbium, we describe the atom as follows. The 28electron innermost core, $1 s^{2} 2 s^{2} 2 p^{6} 3 s^{2} 3 p^{6} 3 d^{10}$, is replaced by vGRPP, i.e. the small-core RPP version is assumed. The remaining (valence and outer-core) 42 electrons, which correspond to the $4 s^{2} 4 p^{6} 4 d^{10} 4 f^{14} 5 s^{2} 5 p^{6} 6 s^{2} 5 d^{0}$ configuration, are explicitly included into the calculations. We call this RPP version as 42ve-RPP below.

For fluoride atoms nearest to the central $\mathrm{Yb}$, we use the all-electron model.

\subsection{Theoretical study of chemical shifts of $X$-ray emission lines}

The calculation of chemical shifts of X-ray emission lines is usually a challenging problem for modern $a b$ initio methods when studying compounds containing heavy atoms including transition metals, actinides, and lanthanides. The theoretical investigation of such systems should take into account both the relativistic and correlation effects very accurately. In references [4] and [5], a method of evaluating chemical shifts of $\mathrm{X}$-ray emission lines is described, which is applicable to such kind of systems. It is based on the relativistic pseudopotential model and one-center restoration approach [6] to recover a proper electronic structure in heavy-atom cores after the pseudopotential simulation of chemical compounds. An expression for chemical shift as a difference between mean values of certain one-electron effective operator is proposed there. It corresponds (in simple model cases but not generally) to the difference between the orbital energies of the initial and final shells in the compounds. It follows from [4] that the operator related to a XES chemical shifts is concentrated in the atomic core region. On the other hand, the main non-vanishing contribution to the matrix elements of this operator is due to the valence electrons, whereas the contributions from closed innercore shells compensate each other. It has been shown by our group (see Ref. [6] and references therein) that the procedure of evaluating such characteristics as XES chemical shifts can be significantly simplified by splitting the calculation into two steps. At the first step, the electronic correlation for valence electrons (and outer-core electrons for better accuracy) is taken into account in a molecular calculation using some method of electronic structure treatment (such as coupled cluster, configuration interaction, DFT, etc.). In turn, the core electrons (inner-core in our case with $\mathrm{Yb}$ ) are excluded from the calculations with vGRPP [8], [9] so that an accurate electronic structure of valence region is evaluated most economically. Since the inner-core parts of the valence one-electron "pseudo-wave functions" are smoothed within the vGRPP method; they have to be recovered using some core-restoration method [6] at the second step. The non-variation restoration technique, employed in the present work, is based on approximate proportionality of valence and virtual spinors in the inner-core region of heavy atoms (see, e.g., Ref. [9] for details). The two-step approach has been recently used in references [12]-[16] for calculating various characteristics, such as hyperfine structure constants, etc. in molecules and atoms. The two-step method has been also extended to the case of crystals in Ref. [17]. Comparison of the two-step approach (in which the inner-core electrons are treated as frozen) with the all-electron treatment was made in papers [17-19], where an agreement on the level of accuracy of the used correlation methods is attained.

\subsection{Modeling of the crystal structure}

In our study, the experimental structural data for the crystals are used. The $\mathrm{YbF}_{2}$ crystal has $\mathrm{Fm}-3 \mathrm{~m}$ (225) space group and its cell parameters are $\mathrm{a}=0.55991 \mathrm{~nm}, \mathrm{~b}=0.55991 \mathrm{~nm}, \mathrm{c}=0.55991 \mathrm{~nm}$, $\alpha=90^{\circ}, \beta=90^{\circ}, \gamma=90^{\circ}$ [20], and the $\mathrm{YbF}_{3}$ crystal has Pnma (62) space group and the cell parameters are $\mathrm{a}=0.6218 \mathrm{~nm}, \mathrm{~b}=0.6785 \mathrm{~nm}, \mathrm{c}=0.4431 \mathrm{~nm}, \alpha=90^{\circ}$, $\beta=90^{\circ}, \gamma=90^{\circ}[21]$.

We model the embedded cluster for structures of $\mathrm{YbF}_{2}$ and $\mathrm{YbF}_{3}$ crystals as follows. An ytterbium atom is considered as an origin in the clusters and its nearest coordination spheres are reproduced in accord to the positions of corresponding atoms in the unit cell.

Thus for the cluster simulation of the $\mathrm{YbF}_{2}$ crystal, eight fluorine atoms are located around the central ytterbium at a distance of $0.2424 \mathrm{~nm}$. They form a cube which is centered at the origin of our cluster. These fluoride atoms constitute the first coordination sphere. It is important to reproduce the electronic structure of $\mathrm{YbF}_{2}$ within at least the "minimal cluster", $\mathrm{YbF}_{8}$, around $\mathrm{Yb}$ in order to evaluate XES chemical shifts on $\mathrm{Yb}$ rather accurately. Note also that for a cluster of such size ( $<10$ atoms) most reliable wave-function based correlation methods can be applied in principle.

Further, at $0.3959 \mathrm{~nm}$ distance from the center, twelve $\mathrm{Yb}$ atoms are located (which constitute the second coordination sphere). The next (third) coordination sphere consists of twenty four fluorine atoms at a distance of $0.4643 \mathrm{~nm}$. We replace these atoms by pseudopotentials to ensure the proper embedding of the above minimal cluster into the $\mathrm{YbF}_{2}$ crystal. Note that all these coordination spheres form 
V.M. Shakhova et al., Chemical shifts of x-ray emission spectra..., Rad. Applic., 2017, 2, 3, 169-174

highly symmetric substructures (see Figure 1). Below, we designate this extended cluster as $\mathrm{YbF}_{8} \mathrm{Yb}_{12} \mathrm{~F}_{24}$.

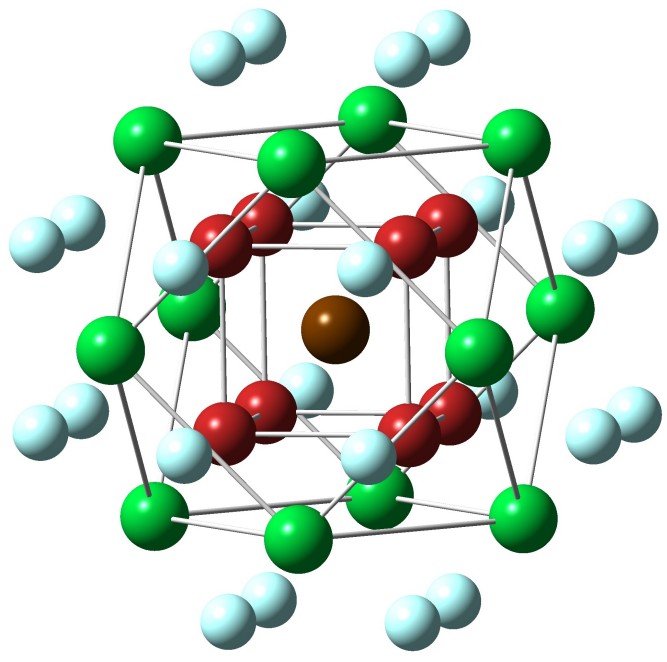

Figure 1. Structure of $\mathrm{YbF}_{8} \mathrm{Yb}_{12} \mathrm{~F}_{24}$ cluster. The central $\mathrm{Yb}$ atom is highlighted in brown, $\mathrm{F}$ atoms of the first coordination sphere are red, $\mathrm{Yb}$ atoms of the second coordination sphere are green, and $\mathrm{F}$ atoms of the third coordination sphere are blue

Table 1. Distances from the center ytterbium atom for $\mathrm{Yb}$ and $\mathrm{F}$ atoms in the $\mathrm{YbF}_{8} \mathrm{Yb}_{12} \mathrm{~F}_{24}$ and $\mathrm{YbF}_{9} \mathrm{Yb}_{12} \mathrm{~F}_{24}$ clusters, $\mathrm{nm}$

\begin{tabular}{|c|c|c|c|}
\hline \multirow[t]{2}{*}{$\begin{array}{c}\text { Cluster } \\
\mathrm{YbF}_{8} \mathrm{Yb}_{12} \mathrm{~F}_{24}\end{array}$} & $\begin{array}{c}\text { First coord. } \\
\text { sphere } \\
\text { Yb-F }\end{array}$ & $\begin{array}{c}\text { Second } \\
\text { coord. sphere } \\
\text { Yb-Yb }\end{array}$ & $\begin{array}{c}\text { Third coord. } \\
\text { sphere } \\
\text { Yb-F }\end{array}$ \\
\hline & 0.2424 & 0.3959 & 0.4643 \\
\hline \multirow{21}{*}{$\begin{array}{c}\text { Cluster } \\
\mathrm{YbF}_{9} \mathrm{Yb}_{12} \mathrm{~F}_{24}\end{array}$} & $\begin{array}{l}\text { First coord. } \\
\text { region }\end{array}$ & \multicolumn{2}{|c|}{ Second coord. region } \\
\hline & $\mathrm{Yb}-\mathrm{F}$ & $\mathrm{Yb}-\mathrm{Yb}$ & $\mathrm{Yb}-\mathrm{F}$ \\
\hline & & & $\begin{array}{l}0.3527 \\
0.3527 \\
0.3783 \\
0.3783 \\
0.4059 \\
0.4059\end{array}$ \\
\hline & & 0.3562 & 0.4157 \\
\hline & 0.2222 & 0.3562 & 0.4190 \\
\hline & 0.2246 & 0.3803 & 0.4190 \\
\hline & 0.2264 & 0.3803 & 0.4190 \\
\hline & 0.2264 & 0.4112 & 0.4190 \\
\hline & 0.2268 & 0.4112 & 0.4310 \\
\hline & 0.2268 & 0.4306 & 0.4352 \\
\hline & 0.2289 & 0.4306 & 0.4352 \\
\hline & 0.2289 & 0.4306 & 0.4424 \\
\hline & 0.2612 & 0.4306 & 0.4424 \\
\hline & & 0.4431 & 0.4506 \\
\hline & & 0.4431 & 0.4506 \\
\hline & & & 0.4562 \\
\hline & & & 0.4562 \\
\hline & & & 0.4657 \\
\hline & & & 0.4657 \\
\hline & & & 0.4694 \\
\hline & & & 0.4694 \\
\hline
\end{tabular}

The embedded cluster for the $\mathrm{YbF}_{3}$ crystal has a more complicated structure. If we look at the area around the central atom of the order of $0.46 \mathrm{~nm}$ (spatially- and size-balanced with the $\mathrm{YbF}_{8} \mathrm{Yb}_{12} \mathrm{~F}_{24}$ cluster simulating the $\mathrm{YbF}_{2}$ crystal), we find there 33 fluorine atoms and 12 ytterbium atoms. Below we designate this cluster as $\mathrm{YbF}_{9} \mathrm{Yb}_{12} \mathrm{~F}_{24}$. In Table 1, the distances from the center for the corresponding atoms are listed.

The first coordination sphere of $\mathrm{YbF}_{9} \mathrm{Yb}_{12} \mathrm{~F}_{24}$ cluster consists of nine fluorine atoms. It corresponds to the "minimal cluster" model, $\mathrm{YbF}_{9}$, for the $\mathrm{YbF}_{3}$ crystal. The other atoms in the $\mathrm{YbF}_{9} \mathrm{Yb}_{12} \mathrm{~F}_{24}$ cluster are used to describe embedding of the $\mathrm{YbF}_{9}$ cluster to the crystal. It is finally used to evaluate the XES chemical shifts on the central $\mathrm{Yb}$.

In contrast to the $\mathrm{YbF}_{8} \mathrm{Yb}_{12} \mathrm{~F}_{24}$ case, we cannot uniquely identify the second and third coordination spheres (as those for atoms of the same kind) for the $\mathrm{YbF}_{9} \mathrm{Yb}_{12} \mathrm{~F}_{24}$ cluster. Nevertheless, this is the cluster that is reasonable to use in the simulation of $\mathrm{YbF}_{3}$ crystal for the XES chemical shift evaluation (along with the $\mathrm{YbF}_{8} \mathrm{Yb}_{12} \mathrm{~F}_{24}$ cluster simulating $\mathrm{YbF}_{2}$ ) based on the spatial- and size-balancing arguments. Thus, the second coordination region for $\mathrm{YbF}_{3}$ crystal contains both the ytterbium and fluorine atoms. The structure of the $\mathrm{YbF}_{9} \mathrm{Yb}_{12} \mathrm{~F}_{24}$ cluster in the projections onto $\mathrm{XY}$ plane, XZ plane, YZ plane are shown in Figure 2.

It should be noted that both the $\mathrm{YbF}_{8} \mathrm{Yb}_{12} \mathrm{~F}_{24}$ and $\mathrm{YbF}_{9} \mathrm{Yb}_{12} \mathrm{~F}_{24}$ clusters should be electrically neutral to avoid the unphysical charge transfer and convergence problems [22]. The simplest way to satisfy this is to model the same atoms in different coordination spheres by different ways.

As noticed above, the central ytterbium atom in the $\mathrm{YbF}_{8} \mathrm{Yb}_{12} \mathrm{~F}_{24}$ cluster is described by the 42ve-RPP. The atom has an oxidation state +2 . The basis sets (10s1op7d7f2g)/ [9s8p5d4f2g] for ytterbium compatible with the vGRPP was generated aimed for the relDFT calculations of $\mathrm{Yb}$ compounds.

Eight fluorine atoms of the first coordination sphere are included in the calculations with all electrons treated explicitly and aug-cc-pVTZ basis set was used for them [23]. The minimum cluster $\mathrm{YbF}_{8}$ has a formal charge of -6 . Consequently, the remaining atoms of the $\mathrm{YbF}_{8} \mathrm{Yb}_{12} \mathrm{~F}_{24}$ cluster must compensate this charge for electrical neutrality. So we simulate (an average) charge of +1.5 on each of twelve ytterbium atoms from second coordination sphere that roughly corresponds to a partial charge on $\mathrm{Yb}$ in the $\mathrm{YbF}_{2}$ compounds [24]. They are described by the 2ve-RPP (generated for the $\left[\mathrm{Xe} 4 f^{14}\right] 6 s^{0.1} 6 p^{0.1} 5 d^{0.1} 5 f^{0.1} 5 g^{0.1}$ configuration) with the reduced effective nuclear charge (from +2 to +1.5 ) and minimal basis set (4s4p4d) used on these atoms. The fluorine atoms from the third coordination sphere are treated in the form of point-nuclei with effective charges of -0.5 (to satisfy the electroneutrality of the cluster) with minimal basis set (3s)/[2s] on them which corresponds to $3-21 \mathrm{G}$ basis set for $\mathrm{H}$ atom [25]. 


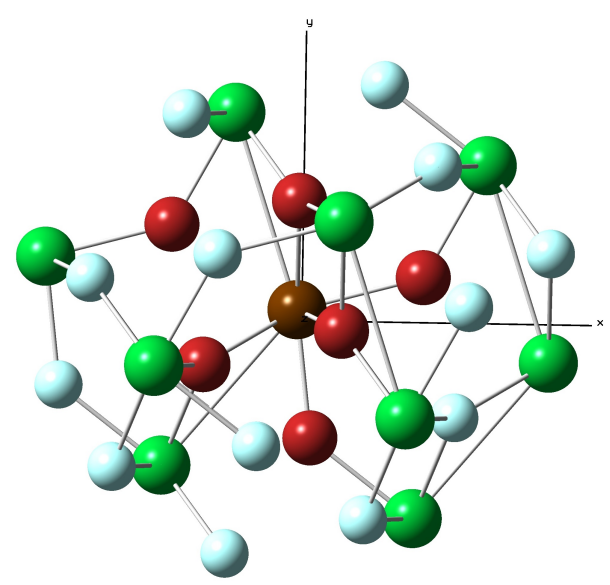

Figure 2a. Structure of $\mathrm{YbF}_{9} \mathrm{Yb}_{12} \mathrm{~F}_{24}$ cluster projected on the $\mathrm{XY}$ plane. The designations are equivalent to those in Figure 1

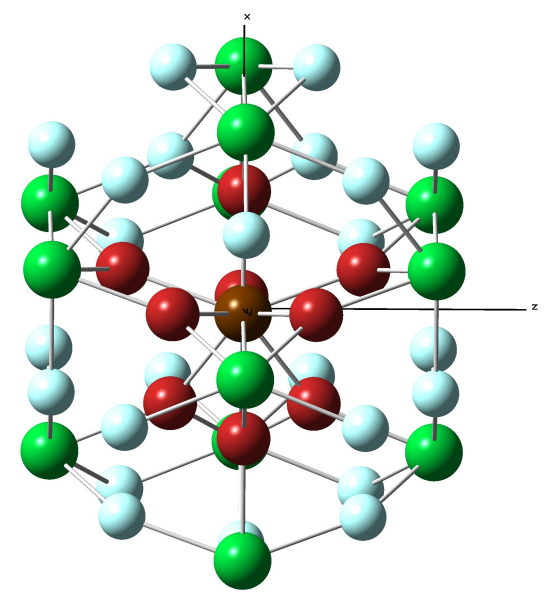

Figure 2b. Structure of $\mathrm{YbF}_{9} \mathrm{Yb}_{12} \mathrm{~F}_{24}$ cluster projected on the $\mathrm{XZ}$ plane. The designations are equivalent to those in Figure 1

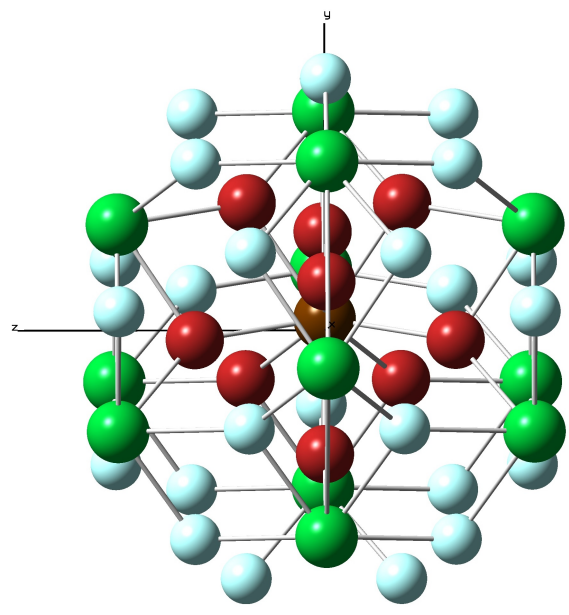

Figure 2c. Structure of $\mathrm{YbF}_{9} \mathrm{Yb}_{12} \mathrm{~F}_{24}$ cluster projected on the $\mathrm{YZ}$ plane. The designations are equivalent to those in Figure 1

Now we consider the embedded cluster simulation of $\mathrm{YbF}_{3}$. In the case of $\mathrm{YbF}_{9} \mathrm{Yb}_{12} \mathrm{~F}_{24}$ cluster, we use particularly generated pseudopotential and basis set for $\mathrm{Yb}$ atoms from the second coordination region and different effective charge on $\mathrm{Yb}$ and $\mathrm{F}$ atoms from this region. The cluster of minimal size, $\mathrm{YbF}_{9}$, has a total charge -6 like a minimal cluster $\mathrm{YbF}_{8}$. The charges on twelve ytterbium atoms are chosen as +2.5 , which roughly (in average) correspond to partial charges on $\mathrm{Yb}$ in the $\mathrm{YbF}_{3}$ compounds [24]. These atoms are described by 3ve-RPP for ytterbium (generated for the $\left[\mathrm{Xe} 4 f^{13}\right] 5 d^{0.6} 6 s^{0.1} 6 p^{0.1}$ configuration with the open $4 f$ shell treated as a core shell, i.e. excluded from the explicit treatment). The effective nuclear charge was correspondingly reduced from +3 to +2.5 . The basis set (7s5p3d) on these atoms was used. The twenty four fluorine atoms have a charge -1 and thus the electroneutrality of the cluster is satisfied.

\section{RESULTS AND DISCUSSIONS}

The calculations are performed within relHF and relDFT using the hybrid exchange-correlation functional PBEo that comprise $25 \%$ of the Hartree-Fock exchange [26], [27]. The computer code [28] was used.

The non-variation restoration code developed in references [14], [17], [29], [30] was used to obtain the correct four-component electronic wave function near the $\mathrm{Yb}$ nucleus. Chemical shifts of $\mathrm{K}_{a_{1}, 2}$ lines were calculated at experimental structural data [20], [21] by the method described in [4], assuming the core restoration radius $R_{c}=0.55$ a.u. (see Table 2 ). The experimental value for the chemical shift of $\mathrm{K}_{\alpha}$ lines for the $\mathrm{YbF}_{3}$ crystal with respect to the $\mathrm{YbF}_{2}$ one was taken from Ref. [31] (the $K_{\alpha 1}$ and $K_{\alpha 2}$ lines were not resolved in the experiment).

Table 2. Computed chemical shifts of $\mathrm{K}_{\alpha_{1}}$ and $\mathrm{K}_{\mathrm{a}_{2}}$ lines for $\mathrm{YbF}_{9} \mathrm{Yb}_{12} \mathrm{~F}_{24}$ cluster with respect to $\mathrm{YbF}_{8} \mathrm{Yb}_{12} \mathrm{~F}_{24}$ cluster, meV

\begin{tabular}{|c|c|c|c|}
\hline Method & $\mathrm{K}_{\alpha 1}$ & $\mathrm{~K}_{\mathrm{a} 2}$ & $\begin{array}{c}\text { Weighted } \\
\text { mean } \mathrm{K}_{\alpha}^{\text {theor }}\end{array}$ \\
\hline $\begin{array}{c}\text { relHF } \\
\text { relDFT/PBEo }\end{array}$ & 602 & 627 & 677 \\
\hline Exp. [31] & \multicolumn{3}{|c|}{$557 \pm 27$} \\
\hline
\end{tabular}

One can see that the PBEo values are in a better agreement with the experimental data than the RHF ones that indicates the importance of accounting for correlation effects.

In order to compare our values with the experimental one, we use a weighted mean $\mathrm{K}_{a}{ }^{\text {theor }}=\left(2 \mathrm{~K}_{\alpha 1}+\mathrm{K}_{\alpha 2}\right) / 3=605 \mathrm{meV}$ (relDFT/PBEo), that agrees with the experiment within ca. 10\%. The relHF method yields $\mathrm{K}_{a}^{\text {theor }}=677 \mathrm{meV}$ overestimating the experimental datum by more than $20 \%$.

\section{CONCLUSIONS}

The environments of $\mathrm{Yb}$ atoms in $\mathrm{YbF}_{2}$ and $\mathrm{YbF}_{3}$ crystals are simulated within a cluster model using embedding potential technique. Both RPPs and embedding potentials for $\mathrm{Yb}$ and $\mathrm{F}$ atoms were constructed. The cluster model allows one to use different levels of correlation treatment, from DFT to 
V.M. Shakhova et al., Chemical shifts of x-ray emission spectra..., Rad. Applic., 2017, 2, 3, 169-174

the coupled cluster one, and flexible basis sets for better control of the computational accuracy. The application of the latter is planed in further studies.

The chemical shifts for $K_{a 1}$ and $K_{a 2}$ lines in the $\mathrm{YbF}_{9} \mathrm{Yb}_{12} \mathrm{~F}_{24}$ cluster with respect to $\mathrm{YbF}_{8} \mathrm{Yb}_{12} \mathrm{~F}_{24}$ were calculated using the relHF method and relDFT with the hybrid functional PBEo.

The weighted mean of computed PBEo chemical shifts for $\mathrm{K}_{\alpha}$ lines agree within $10 \%$ with experimental datum that we consider as a good result, whereas the neglect of electron correlations within the relHF approximation leads to the overestimation of the chemical shift by more than $20 \%$.

Acknowledgement: We are indebted to Prof. C. van Wuellen for providing us with his relativistic DFT code [26]. This work is supported by the Russian Science Foundation (grant no. 14-31-OOO22).

\section{REFERENCES}

1. K. Siegbahn, "From X-Ray to Electron Spectroscopy," in Lecture Notes in Physics: Nishina Memorial Lectures, vol. 746, Osaka, Japan: Springer, 2009, ch. 8, pp. 137 228.

DOI: $10.1007 / 978-4-431-77056-5 \_8$

2. O. I. Sumbaev, "Shift of K X-ray lines associated with valency change and with isomorphous phase transitions in rare earths," Phys. Usp., vol. 21, no. 2, pp. $141-154$, 1978. DOI: 10.1070/PU1978v021no2ABEHoo5519

3. R. I. Karaziya, A. I. Udris, D. V. Grabauskas, "Use of the Chemical Shifts of Electron Levels in the Study of the Distribution of the Effective Charges of Atoms in Compounds," J. Struct. Chem., vol. 18, no. 4, pp. 520 $525,1977$. DOI: $10.1007 / \mathrm{BFoO} 745283$

4. Y. V. Lomachuk, A. V. Titov, "Method for Evaluating Chemical Shifts of X-ray Emission Lines in Molecules and Solids," Phys. Rev. A, vol. 88, 062511, 2013. DOI: 10.1103/PhysRevA.88.062511

5. A. V. Titov, Y. V. Lomachuk and L. V. Skripnikov, "Concept of effective states of atoms in compounds to describe properties determined by the densities of valence electrons in atomic cores," Phys. Rev. A, vol. 90, 052522, Nov. 2014. DOI: 10.1103/PhysRevA.90.052522

6. A. V. Titov, N. S. Mosyagin, A. N. Petrov, T. A. Isaev, D. P. DeMille, "Study of P,T-parity violation effects in polar heavy-atom molecules," in Progress in Theoretical Chemistry and Physics: Recent Advances in the Theory of Chemical and Physical Systems, vol. 15, J.-P. Julien, J. Maruani, D. Mayou, S. Wilson, G. Delgado-Barrio, Eds., Dordrecht, Netherlands: Springer, 2006, ch, 12, pp. 253-283.

DOI: $10.1007 / 1-4020-4528-X \_12$

7. P. A. Christiansen, Y. S. Lee and K. S. Pitzer, "Improved ab initio effective core potentials for molecular calculations," J. Chem. Phys., vol. 71, no. 11, pp. 44454450, 1979.

DOI: $10.1063 / 1.438197$

8. N. S. Mosyagin, A. V. Zaitsevskii and A.V. Titov, "Shape-consistent relativistic effective potentials of small atomic cores, international review of atomic and molecular physics," Int. Rev. At. Mol. Phys., vol. 1, no. 1 , pp. $63-72,2010$.

Retrieved from:

https://pdfs.semanticscholar.org/82f5/65187ec338407 439d7cb111e58e53adfc19c.pdf
Retrieved on: Aug. 5, 2017

9. A. V. Titov and N. S. Mosyagin, "Generalized relativistic effective core potential: Theoretical grounds," Int. J. Quantum Chem., vol. 71, no. 5, pp. 359 - 401, 1999. DOI: 10.1002/(SICI)1097-461X(1999)71:5<359::AIDQUA1>3.o.CO;2-U

10. N. S. Mosyagin, A. V. Zaitsevskii, L. V. Skripnikov, A. V. Titov, "Generalized relativistic effective core potentials for actinides," Int. J. Quantum Chem., vol. 116, no. 4, pp. $301-315$, Feb. 2016. DOI: $10.1002 /$ qua.24978

11. A. V. Titov and N. S. Mosyagin, "Generalized relativistic effective core potential method: Theory and calculations," Russ. J. Phys. Chem., vol. 74, suppl. 2, pp. S376 - S387, 2000.

Retrieved from:

https://arxiv.org/pdf/physics/0008160.pdf Retrieved on: Aug. 5, 2017

12. L. V. Skripnikov, A. N. Petrov, A. V. Titov, N.S. Mosyagin, "Electron electric dipole moment: Relativistic correlation calculations of the P,T-violation effecting the $3 \Delta_{3}$ state of $\mathrm{PtH}^{+}$, , Phys. Rev. A, vol. 8o, no. 6, 060501(R), Dec. 2009. DOI: 10.1103/PhysRevA.80.060501

13. L. V. Skripnikov, A.V. Titov, A. N. Petrov, N. S. Mosyagin, O. P. Sushkov, "Enhancement of the electron electric dipole moment in $\mathrm{Eu}^{2+}$," Phys. Rev. A, vol. 84, no. 2, 022505, Aug. 2011.

DOI: 10.1103/PhysRevA.84.022505

14. A. N. Petrov, "Hyperfine and Zeeman interactions of the a(1) $\left[{ }^{3} \sigma_{1}+\right]$ state of PbO," Phys. Rev. A, vol. 83 , no. 2, 024502, Feb. 2011.

DOI: $10.1103 /$ PhysRevA.83.024502

15. J. Lee et al., "Optical spectroscopy of tungsten carbide for uncertainty analysis in electron electric dipole moment search," Phys. Rev. A, vol. 87, no. 2, 022516 , Feb. 2013.

DOI: 10.1103/PhysRevA.87.022516

16. A. N. Petrov, L. V. Skripnikov, A. V. Titov, R. J. Mawhorter, "Centrifugal correction to hyperfine structure constants in the ground state of lead monofluoride," Phys. Rev. A, vol. 88, no. 1, 010501(R), Jul. 2013.

DOI: 10.1103/PhysRevA.88.010501

17. L. V. Skripnikov, A. V. Titov, "LCAO-based theoretical study of $\mathrm{PbTiO}_{3}$ crystal to search for parity and time reversal violating interaction in solids," J. Chem. Phys. vol. 145, no. 5, 054115, Aug. 2016 DOI: $10.1063 / 1.4959973$

18. L. V. Skripnikov, "Combined 4-component and relativistic pseudopotential study of ThO for the electron electric dipole moment search," J. Chem. Phys., vol. 145, no. 21, 214301, Dec. 2016. DOI: $10.1063 / 1.4968229$ PMid: 28799403

19. L. V. Skripnikov, A. D. Kudashov, A. N. Petrov, A. V. Titov, "Search for parity- and time-and-parity-violation effects in lead monofluoride (PbF): Ab initio molecular study," Phys. Rev. A, vol. 90, no. 6, 064501, Dec. 2014. DOI: 10.1103/PhysRevA.90.064501

20. T. Petzel, O. Greis, "The vaporization behavior of ytterbium(III) fluoride and ytterbium(II) fluoride," J. Less-Common Met., vol. 46, no. 2, pp. 197 - 207, May 1976. DOI: 10.1016/0022-5088(76)90210-1

21. B. V. Bukvetskii, L. S. Garashina, "Crystal-Chemical Investigation of the Orthorhombic Trifluorides of Samarium, Holmium, and Ytterbium," Sov. J. Coord. Chem., vol. 3, pp. 791 - 795, 1977.

22. I. V. Abarenkov, M. A. Boyko, "Wave-Function-Based Embedding Potential for Ion-Covalent Crystals," Int. $J$ Quantum Chem., vol. 116, no. 3, pp. 211 - 236, Feb. 2016. 
V.M. Shakhova et al., Chemical shifts of x-ray emission spectra..., Rad. Applic., 2017, 2, 3, 169-174

DOI: $10.1002 /$ qua.25041

23. R. A. Kendall, T. H. Dunning, Jr. and R. J. Harrison, "Electron affinities of the first-row atoms revisited: systematic basis sets and wave functions," J. Chem. Phys., vol. 96, no. 9, 6796, May 1992.

DOI: $10.1063 / 1.462569$

24. S. G. Semenov, M. E. Bedrina, A. V. Titov, "Quantumchemical Study of Ytterbium Fluorides and of Complex $\mathrm{F}_{2} \mathrm{YbF}_{2} \mathrm{CeF}_{2}$," Russ. J. Gen. Chem., vol. 86, no. 6, pp. $1215-1220$, Jun. 2016

DOI: $10.1134 / \mathrm{S} 1070363216060013$

25. J. S. Binkley, J. A. Pople, W. J. Hehre, "Self-Consistent Molecular Orbital Methods. 21. Small Split-Valence Basis Sets for First-Row Elements," J. Am. Chem. Soc., vol. 102, no. 3, pp. 939 - 947, Jan .1980. DOI: $10.1021 /$ jaoo523aoo8

26. J. P. Perdew, K. Burke, M. Ernzerhof, "Generalized gradient approximation made simple," Phys. Rev. Lett., vol. 77 , no. 18 , pp. $3865-3868,1996$

DOI: 10.1103/PhysRevLett.77.3865 PMid: 10062328

27. C. Adamo, V. Barone, "Toward reliable density functional methods without adjustable parameters: The PBEo model," J. Chem. Phys., vol. 110, no. 13, pp. 6158 - 6170, Apr. 1999.

DOI: $10.1063 / 1.478522$
28. C. van Wuellen, "A quasirelativistic two-component density functional and Hartree-Fock program," Z. Phys. Chem., vol. 224, no. 3-4, pp. $413-426,2010$. DOI: $10.1524 /$ zpch.2010.6114

29. L. V. Skripnikov, A. V. Titov, "Theoretical study of $\mathrm{ThF}^{+}$ in the search for t,p-violation effects: Effective state of a Th atom in $\mathrm{ThF}^{+}$and ThO compounds," Phys. Rev. A, vol. 91, no. 4, 042504, Apr. 2015. DOI: 10.1103/PhysRevA.91.042504

30. L. V. Skripnikov, A. N. Petrov and A. V. Titov, "Communication: Theoretical study of ThO for the electron electric dipole moment search," J. Chem. Phys., vol. 139, no. 22, 221103, Dec. 2013

DOI: $10.1063 / 1.4843955$

PMid: 24329049

31. В.А. Шабуров и др, “Состояния промежуточной валентности иттербия в интерметаллических соединениях,” т. 24, но. 1, стр. 263 - 265, 1982. (V. A. Shaburov et al., "State of the intermediate valence ytterbium in intermetallic compounds," Phys. Solid State, vol. 24, no. 1, pp. 263-265, 1982.) 